\title{
PREDICTION OF ROTARY FILES LIFE SPAN USING FOUR METHODS
}

\author{
Yasser F Gomaa* and Magdy Mohamad Ali**
}

\begin{abstract}
This study was designed to find a suitable method for estimating and predicting the average life span of rotary files after simulated clinical use.

Materials and methods: We used One Shape rotary file system. A total of 120 mesiobuccal root canals from lower 1st molars were used with medium curvatures in the range of 20-40 degrees. All canals were prepared at torque of $4 \mathrm{~N} / \mathrm{cm}^{2}$ and $350 \mathrm{rpm}$. Files were subjected to autoclaving after preparing each 3 canals. Files were evaluated for deformation by four methods; magnifying loupe, DOM, SEM and atomic force microscope at the base line, after preparing 3,6,9 and 12 canals. Scores were recorded. We used a modified form of the classification of Chekka et al to evaluate the deformation of files.
\end{abstract}

Results: Some of -as received- files showed some sort of deformation evidenced by M.L and DOM and this was doubled when evaluated using SEM or AFM. All files developed more deformation after preparing more canals. The score of deformation ranged between 1-67\%. No files exhibited fracture before preparing 9 canals. The score of deformation was in direct relation to the number of prepared canals. The significant differences in the tools of evaluation were not significant until preparing nine canals.

Conclusion the results reflect the importance of the loupe in detecting major deformations and the DOM in detecting the minor deformations. It also gives a notation about the importance of regular check up of the files after use and using visual aids specially ones with high magnification powers as the DOM. There were significant difference of the results of the evaluation methods after preparing nine canals with atomic force microscope the first detection method followed by SEM, DOM and finally M L the implication of this finding is M.L. and DOM are satisfactory clinical means of evaluation of the files and stating the time of discarding.

* Assistant Professor, Dental Materials Department, Faculty of Dentistry, Minia University, Egypt.

** Assistant Professor, Endodontics Department, Faculty of Dentistry, Minia University, Egypt. 


\section{INTRODUCTION}

The cleaning ability of any root canal instrument is of importance for the outcome of root canal treatment ${ }^{[1]}$. Since the introduction of highly flexible alloy to endodontics, Nikel Titanium (Ni-Ti) hand files and rotary instruments have achieved widespread popularity because of superiority of rotary(Ni-Ti) in preparing curved root canals ${ }^{[2]}$. Rotary Ni-Ti root canal instruments have become an important part of endodontic armamentarium. Despite these advantages, NiTi instruments appear to have a high risk of separation, mainly because of fatigue and torsional shear stresses. This happens when the instrument rotate inside a curved canal and is subjected to an excessive number of tension-compression strain cycles in the region of maximum root canal curvature ${ }^{[3,4]}$. Some factors have been implicated in the separation of Ni-Ti endodontic instruments, amongst are: operator proficiency, method of use, rotational speed, design of the instrument, and number of sterilization cycles ${ }^{[5,7]}$. A new concept of single use instrumentation is that a single file is to be used in a full clockwise rotation. One Shape rotary file belongs to this group of single-file system. In the past years, the reciprocating motion of the NiTi rotary instrument has been shown to decrease the impact of cyclic fatigue compared with rotational motion ${ }^{[8,10]}$. Therefore, it has been recently proposed that the single-file shaping technique may simplify instrumentation protocols and avoid the risk of cross-contamination. However, single use, mean that the same instrument can be used in 3-4 root canals. Several studies have been conducted to examine and evaluate the $\mathrm{Ni}$ - $\mathrm{Ti}$ Instruments deformation and fracture using scanning electron microscope (SEM) and Stereo-microscope ${ }^{[11,12]}$. Accordingly, we intended to reproduce the complex clinical situation affecting the stresses and strains imposed on the instrument. Hence, we can evaluate the cumulative effect on One shape rotary $\mathrm{Ni}$ Ti system using magnifying loupe (ML), dental operating microscope[DOM,] SEM and Atomic force microscope (AFM) after each use for 3,6,9 and 12 root canals preparation although the One shape rotary file belongs to single use instrumentation system many clinicians used it for preparing more than 3 root canals after sterilization for economic purpose $^{[13]}$.

\section{MATERIALS AND METHODS}

One hundred and twenty mesiobuccal root canals obtained from freshly extracted human lower first molars were used (ethical protocol of the Faculty of Dentistry, Minia University was followed). The teeth were stored in $0.5 \%$ Sodium Azide in fresh water until use. Inclusion criteria were: patients' age ranged from 20-60 years, no perforations and no previous history of endodontic preparation. Exclusion criteria include straight or severely curved canals. Root canals of the chosen teeth were measured to have an average medium curvature ranging from $20^{\circ}$ to $40^{\circ}$ according to the Schneider method ${ }^{[14]}$. The selected teeth were decapitated using a diamond disk and the mesial root was separated from the distal root. The mesial roots were cut coronally to establish $14 \mathrm{~mm}$ length. The root canal length was determined by inserting \# $10 \mathrm{~K}$ - files into the canal until the file was seen from the apical foramen and then $0.5 \mathrm{~mm}$ was reduced from this length to establish the working length. Each root was fixed in a ring filled with pink self cured acrylic resin (Acrostone, Egypt).

Ten set from One Shape rotary files system( Micro Mega, Besancon, France) were used. Mesiobuccal root canals were mechanically prepared by using One Shape Ni-Ti rotary instruments. Each file was mounted on the Endo Mate DT motor (NSK, Kanuma, Japan) set at $350 \mathrm{rpm}$ and $4 \mathrm{~N} / \mathrm{cm}^{2}$ torque with a 16:1 contra angle in clockwise rotation. All manufacturer's recommendations were followed in the preparations. Sodium hypochlorite $(2.5 \%)$ was 
used as an irrigating solution $2 \mathrm{ml}$ after each file entering the canal \& each instrument was lubricated with Glyde File Prep (Dentsply).The coronal 4mm of the mesiobuccal root canals was mechanically prepared with Gats Glidden drills size 2 and 3. Root canal preparation was completed with One Shape file with tip diameter 25 taper 0.06 to complete apical preparation. After each root canal instrumentation, the files were scrubbed under running water \& then introduced in the ultrasonic cleaner for 10 minutes cycle and Autoclaved for 15 minutes under 2 bar pressure.

Before use the assigned files were inspected by dental loupe (Keeler Ltd, Windsor, Berkshire, UK) at 6x magnification and Dental Operating Microscope (DOM) ZIES-Germany) at magnification 16x and snap shots were taken from the computer connected to the DO, Scanning electron microscope SEM. (Quanta 250 FEG, Netherlands with accelerating voltage $30 \mathrm{~K} . \mathrm{V}$ and magnification1000x) and atomic force microscope(AFM) which was operated in contact mode using non-conductive silicon nitride probe (Bruker, MLCT-MT-A, USA) using proscan software for controlling the scan parameters and IP 2,1 software for image analysis and also after preparation of 3,6,9 and12 canals. For evaluation and rating of file defects, We used a modified form of the classification of Chekka et al, 2012, ${ }^{[15]}$ to evaluate the deformation of files. The defects caused by torsional fatigue were divided to two groups depending on the severity of the defect as follows:

\section{1- Defects indicating mild damage}

a) Bent instrument/tip

b) Stretching /straightening of twist contour

c) Slight increase of the surface roughness (up to double initial roughness) as indicated by AFM

d) Cutting edge dented

\section{2- Defects indicating severe damage:}

1- Partial reverse twisting

2- Severe increase of the surface roughness (more than double initial roughness) as indicated by AFM

3- Partial reverse twisting

4- Change in length $(\mathrm{mm})$

5- Fracture of the file

And we recorded the defects in a score as follows:

0 - No deformation

1- Bent instrument/tip

2- Stretching/straightening of twist contour

3- Slight increase of the surface roughness (up to double initial roughness) as indicated by AFM

4- Cutting edge dented

5- Partial reverse twisting

6- Severe increase of the surface roughness (more than double initial roughness) as indicated by AFM

7- Partial reverse twisting

8- Change in length $(\mathrm{mm})$

9- Fracture of the file

When two types of deformation were present in the same file we record the higher one. Values were recorded as a function of score multiplied to the number of corresponding file divided by 10 (the number of files). Then the sum was recorded. Statistical analysis was performed with SPSS 16.0 (Statistical Package for Scientific Studies) for Windows. Qualitative data were presented as frequencies and percentages. Chi-square (x2) test was used for studying the comparisons between different qualitative variables. Reliability analysis using Cronbach's Alpha was used to measure the agreement between Loupe and Dental Operating Microscope evaluations. The significance level was set at $\mathrm{P} \leq 0.05$. 


\section{RESULTS}

One percent of -as received- files showed some sort of deformation when evaluated by ML or DOM. This percent was doubled when evaluated using SEM or AFM. All files developed more deformation after preparing more canals. The score of deformation ranged between 1-67\%. No files exhibited fracture before preparing 9 canals. The score of deformation was in direct relation to the number of prepared canals. Scores of deformation with different evaluation methods at the base line (as received) and after preparing 3,6,9 and 12 canals are presented in (tables 1 ) and as bar chart in
(Fig. 1) Although the deformation detected by AFM usually precede other examination methods, there was no significant difference between different examination tools after preparing 3 or 6 or 12 canals. The differences were significant after preparing nine canals. Surface roughness increase dramatically after preparing 3 canals. After preparing 9 and 12 canals the difference in evaluation using ML and DOM was not significant. The same insignificant differences were found between SEM and AFM. Most deformations were noticed in the separations occurred predominantly at the D10 to D12 level.

Table (1) percentage of file deformation score at base line and after preparing 3,6,9 and 12 canals:

\begin{tabular}{|c|c|c|c|c|c|}
\hline & Base line & 3 canals & 6canals & 9 canals & 12 canals \\
\hline ML & 1 & 9 & 34 & 48 & 65 \\
\hline DOM & 1 & 2 & 38 & 50 & 61 \\
\hline SEM & 2 & 17 & 43 & 60 & 67 \\
\hline
\end{tabular}

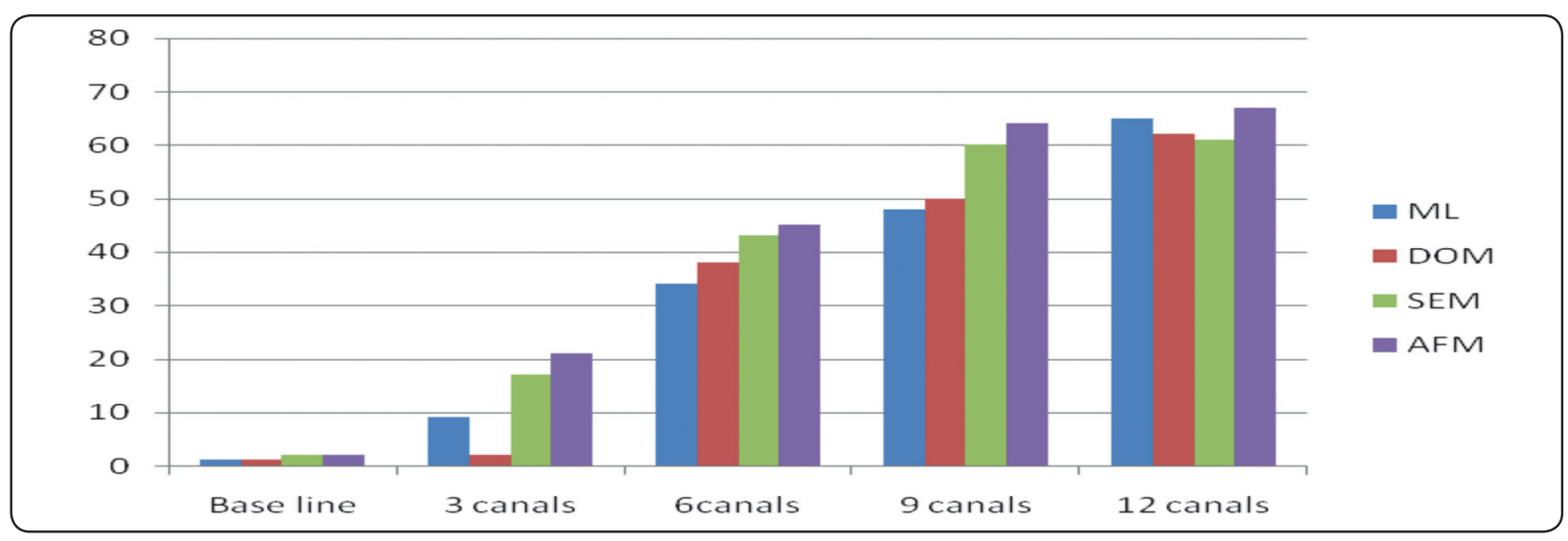

Fig. (1) Bar Chart of \% file deformation score at base line and after preparing 3,6,9 and 12 canals: 


\section{DISCUSSION}

In this study we used one type of files because several factors might affect the failure of files such as type of alloy, diameter of file, its cross section design, etc.$^{[16,17]}$ Accordingly deformations occurring after multiple uses may be due to cumulative effect of the factors at which the instrument were exposed to; including the different root canal parameters, sterilization cycles, number of uses and experience of the operator which is an important affecting factor differing greatly from one operator to the other. Several studies ${ }^{[18,20]}$ have been conducted to examine and evaluate the deformation and fracture using SEM and Stereo-microscope.

Sundaram et al., ${ }^{[21]}$ tested cyclic fatigue resistance of NiTi files after autoclaving cycles and concluded that multiple sterilization did not compromise the mechanical behavior of modern rotary NiTi endodontic instruments.

In the present study we used magnifying aids of clinical significance (loupe, DOM, SEM and AFM). Atomic force microscope is an important evaluating tool for surface roughness detection because it provides easy direct $3 \mathrm{D}$ evaluation of the surface.

The score and classification of our study is important because it rates the deformation in an ascending manner and multiplying corresponding score to the number of affected files. This provide a direct and easy way for the prevalence of deformation, So defects from 0 to 4 might reflect reduction in efficiency but no high possibility of file separation. However scores more than 4 denotes severe reduction in efficiency and possibility of file fracture. The results of this study revealed the possibility of using One shape file up to 9 canals and still in the safe region away from fracture files. But close analysis of the results explain the manufacturer recommendation not to use the file more than once. After preparing 3 canals the percentage of severe deformation was less than $25 \%$. After preparing 6 canals the percentage of severe deformation was more than $40 \%$ which is usually set as a safety percent. Fracture files were noticed after preparing 12 canals.

It was interesting to notice that the evaluation method after preparing up to 6 canals which lie in the less catastrophic changes was not significant from one method to another. After preparing 9 or 12 canals there were significant difference of the results of the evaluation methods; with atomic force microscope the first detection method followed by SEM, DOM and finally M L the implication of this finding is M.L. and DOM are satisfactory clinical means of evaluation of the files and stating the time of discarding it. While SEM and AFM are important for further research regarding metallurgical changes and surface changes and material behavior before failure.

Tripi et al ${ }^{[22]}$ showed that dentin deposits which were created during preparation of root canals adhere tenaciously in surface cracks, Consequently, it may not be possible to remove such deposits from instruments after being used in the root canals of one patient before clinical use on a subsequent one. These lodged dentine chips seem to cause a wedging action on the machining cracks, which may lead to their propagation from localized tensile stress during clinical use and eventually result in instrument fracture .The results reflect the importance of the loupe in detecting major deformations and the DOM in detecting the minor deformations. It also gives a notation about the importance of regular check up of the files after use and using visual aids specially ones with high magnification powers as the DOM. Hence, it will help much in assisting the operator in deformations detection and proper selection of the files to be discarded avoiding its breakage in the patient's mouth. That is why it is the choice of the operator in selecting the suitable package of instruments according to the skills and availability of suitable instruments and visual aids in the operating field. 
Most of the recorded deformed parts of the files were located at the apical one third which goes with previous work of who related this to its locatiion at the maximum curvature of the canal. Thus the in and out pecking movement used in clinical practice makes the segment of the file subjected to maximum fatigue vary continuously, ${ }^{(23,24)}$. However, this study did not include the other variables the file is subjected to during routine clinical usage with its cumulative effects over the life span of the file. Thompson \& Dummer ${ }^{[25]}$; in their work on simulated root canals, they recommended the use of lubricants to decrease the generation of sticky flakes and irrigation for evacuation to prevent clogging the instrument. In the present study the lubricant (Glyde) and the irrigating solution (Sodium hypochlorit $2.5 \%$ ) were used as manufacturer recommendations.

\section{CONCOLUSION}

The results reflect the importance of the loupe in detecting major deformations and the DOM in detecting the minor deformations. It also gives a notation about the importance of regular check up of the files after use and using visual aids specially ones with high magnification powers as the DOM. Atomic force microscope is an important evaluating tool for surface roughness because it provides easy direct $3 \mathrm{D}$ evaluation of the surface.

There were significant difference of the results of the evaluation methods; with atomic force microscope the first detection method followed by SEM, DOM and finally M L the implication of this finding is M.L. and DOM are satisfactory clinical means of evaluation of the files and stating the time of discarding it.

\section{RECOMMENDATION}

Within the limitation of this study we recommend:

a) Follow manufacturer instruction not to use the file for preparing more than 3 canals to avoid dramatic deformation which denote reduction in efficiency occure after preparing 6 canals. b) Avoid using files for preparing more than 9 canals to avoid file separation which was predominant after preparing 9 canals to be within the safe mode

c) SEM and AFM are important for further research regarding metallurgical and surface. changes

\section{REFERENCES}

1. Bertrand MF, Lupi-Pegurier L, Medioni E. Curved molar root canal preparations using Hero 642 rotary NikelTitanium instruments. Int Endodon J 2001; 34:631-6.

2. Walia H, Bronthy Wa, Gerstein H. An initial investigation of the bending and torsional properties of NiTinol root canal files. J Endodon 1988; 14:346-51.

3. Troian CH, So MVR, Pigueiredo JAP. et al. Deformation of $\mathrm{RaCe}$ and $\mathrm{K} 3$ endodontic instruments according to the number of uses. Int Endodon J 2006; 39:1-10.

4. Turpin YL, Chagneau F, Bartier O. et al. Impact of two theoretical cross section on torsional and bending stresses of Nikle-Titanium root canal instruments. J Endodon; $2000 ; 26: 414$.

5. Turpin YL, Chagneau F, Bartier O. et al. Impact of torsional and bending intertia on root canal instruments. J Endodon 2001;27:333

6. Yard GM, Bou Dagher FE, Machtou P. Cyclic fatigue of ProFile rotary instruments after clinical use. Int Endodon J 2000; 33:204.

7. Yard Gm, Kulkarni GK. Failure of ProFile Ni-Tinstruments used by an inexperienced operator under access limitations. Int Endodon J 2002; 35:536-41.

8. Blum JY, Cohen A, Machtou P. Analysis of forces developed during mechanical preparation of extracted teeth using ProFile Ni-Ti rotary instruments. Int Endodon J 1999; 32:24-31.

9. Martin B, Zelada G, varela P. et al. Factors influencing the fracture of Ni-Ti rotary instruments. Int Endodon J 2003; $36: 262-6$

10. Berutti E, Chandussi G, Gaviglio I. et al. A comparative analysis of torsional and bending stresses in two mathematical models of nickel-titanium rotary instruments: ProTaper versus ProFile. J Endodon 2003; 29:15-9.

11. Prutt JP, Clement DJ, Carnes DL. Cyclic fatigue testing of Ni- Ti endodontic instruments. J Endodon 1997; 23:77-85. 
12. Craveiro MC, Guimor M, Buono VT. Fatigue resistance of Engine-Driven Rotary Ni-Ti Endodontic instruments.J Endodon 2002; 28:765-9.

13. Silvaggo J, Hicks ML. Effect of heat sterilization on the torsional properties of rotary Ni-Ti files. J Endodon 1997; 23:731-4.

14. Shneider SW. A comparison of canal preparation in straight \& curved root canals. Oral Surg, Oral Med, Oral Path 1971; 32:271-27.

15. Chekka P, Muralin K, Ratenkar P, Sanjib DA. Do NITI insterumentes show deformation before separation.defect caused by torsional fatigue in hand and rotary NITI insterumentes wich lead to failure during clinical use. 2012;13[6]:867-72.

16. Gambarini G. Cyclic fatigue of nickel-titanium rotary instruments after clinical use with low- and high-torque endodontic motors. J Endod 2001;27:772-4.

17. Grande NM, Plotino G, Pecci R, Bedini R, Malagnino VA, Somma F. Cyclic fatigue resistance and three-dimensional analysis of instruments from two nickel-titaniumrotary systems. Int Endod J 2006;39:755- 63

18. Cheung GSP, Peng B, Bian Z. et al. Defects in ProTaper S1 instruments after clinical use. Fractographic examination. Int Endodon J 2005 ; 38:802-9.
19. Shen Y, cheung GSP, Bian Z. et al. Comparison of defects in ProFile \& ProTaper systems after clinical use. J Endodon 2006; 32:61-5.

20. Sben Y, Winestock E, Chung GS. et al : Defects in NiTi instru- ments after clinical use. Part 4: Electropolished instruments. J Endodon 2009; 35 : 197-201.

21- Sundaram E, SEBEN Y, WINESTOCK F, CHUNG GS. defect in NiTi insteruments after clinical use. patr 4: Electeropolished insteruments. J Endodon 2007; 33:1217-21.

22. Tripi YL, Chagneau F, Bartier O et al. Impact of torsional and bending iterita on root canal instruments. J Endodon 2001; 27:333-6.

23. Parashos P, Gordon I, Messer HH. Factors influencing defects of rotary nickel-titanium endodontic instruments after clinical use. J Endodon 2004; 30: 722-5.

24 Schafer E, Tepel J. Relationship between Design Features of Endodontic Instruments and their Properties. Part 3. Resistance to bending and Fracture. J Endodon 2001;27: 299-303.

25. Thompson SA, Dummer PM. Shaping ability of HERO 642 rotary Nickel Titanium instruments in simulated root canals. Part 1. Int Endodon J 2000; 33: 248-54. 